PROCEEDINGS OF THE

AMERICAN MATHEMATICAL SOCIETY

Volume 138, Number 9, September 2010, Pages 3159-3161

S 0002-9939(10)10461-4

Article electronically published on April 21, 2010

\title{
FIBER PRODUCTS AND CLASS GROUPS OF HYPERELLIPTIC CURVES
}

\author{
JEFFREY D. ACHTER
}

(Communicated by Ken Ono)

\begin{abstract}
Let $\mathbb{F}_{q}$ be a finite field of odd characteristic, and let $N$ be an odd natural number. An explicit fiber product construction shows that if $N$ divides the class number of some quadratic function field over $\mathbb{F}_{q}$, then it does so for infinitely many such function fields.
\end{abstract}

The asymptotic behavior of $\ell$-torsion in class groups $\mathrm{cl}(K)$ of quadratic function fields $K$ over finite fields $\mathbb{F}_{q}$ is now well understood [EVW09, and to a lesser extent it has been apprehended for two decades Ach06, FW89. Nonetheless, there is persistent interest in constructing quadratic function fields with control over the class group (e.g., BJLS08, Pac09 and the references therein). In view of this, the following observation, whose proof is quite explicit, may be of some interest:

Theorem 1. Let $\mathbb{F}_{q}$ be a finite field of odd characteristic. Let $K / \mathbb{F}_{q}$ be a quadratic function field whose genus is at most $(q-3) / 2$. Let $A$ be a finite abelian group of odd order. If $A$ is a subgroup of $\operatorname{cl}(K)$ (and if $K$ is imaginary), then there are infinitely many (imaginary) quadratic function fields $L / \mathbb{F}_{q}$ with $A \subset \operatorname{cl}(L)$.

Note that the field of constants $\mathbb{F}_{q}$ is preserved. Theorem 1 flows readily from a special case of a fiber product construction in GP05, as follows.

Let $\mathbb{F}$ be a field in which 2 is invertible. A quadratic function field $K / \mathbb{F}$ is a quadratic extension of the rational field $\mathbb{F}(t)$. Equivalently, it is the field of rational functions on a hyperelliptic curve, i.e., $K=\mathbb{F}(X)$ for some smooth, projective curve $X / \mathbb{F}$ equipped with an involution $\iota$ such that $X /\langle\iota\rangle$ is isomorphic to $\mathbb{P}^{1}$. Let $g$ be the genus of $X$. Then $X$ is determined by its branch locus $B \subset \mathbb{P}^{1}$, a reduced divisor of degree $2 g+2$. Although $B$ is necessarily defined over $\mathbb{F}$, it may not admit any $\mathbb{F}$-rational points.

Let $t$ be a coordinate on $\mathbb{P}^{1}$, and suppose that $B$ is disjoint from $\{0, \infty\} \subset \mathbb{P}_{t}^{1}$. Then $B$ is the vanishing locus of a squarefree monic polynomial $h(t) \in \mathbb{F}[t]$ of degree $2 g+2$ such that $h(0) \neq 0$, and $K \cong \mathbb{F}(t)[\sqrt{h(t)}]$. Let $\sqrt{B}$ be the inverse image of $B$ under the map $\sigma: \mathbb{P}_{s}^{1} \rightarrow \mathbb{P}_{t}^{1}$ corresponding to $t \mapsto s^{2}$. If $\mathbb{F}$ is algebraically closed and $B=\left\{a_{1}, \cdots, a_{2 g+2}\right\}$, then $\sqrt{B}=\left\{ \pm \sqrt{a_{1}}, \cdots, \pm \sqrt{a_{2 g+2}}\right\}$. In general, since $\sqrt{B}=B \times_{\mathbb{P}_{s}^{1}} \mathbb{P}_{t}^{1}$, if $B$ is defined over $\mathbb{F}$, then so is $\sqrt{B}$.

The field $K / \mathbb{F}$ is called imaginary if it admits a model $\mathbb{F}(u)[\sqrt{f(u)}]$ with $\operatorname{deg} f$ odd; equivalently, the branch locus $B$ contains an $\mathbb{F}$-rational point.

Received by the editors December 18, 2009.

2010 Mathematics Subject Classification. Primary 11R58; Secondary 11R29, 14H05.

The author was partially supported by NSA grant H98230-08-1-0051.

(C)2010 American Mathematical Society Reverts to public domain 28 years from publication 
Lemma 2. Suppose $X / \mathbb{F}$ is a hyperelliptic curve with branch locus $B \subset \mathbb{P}^{1}$ disjoint from $\{0, \infty\}$. Let $Z / \mathbb{F}$ be the hyperelliptic curve with branch locus $\sqrt{B}$. Then

(a) $Z$ is a Galois cover of $X$ with covering group $\mathbb{Z} / 2$;

(b) $\mathbb{F}(Z)=\mathbb{F}(t)\left[\sqrt{h\left(t^{2}\right)}\right]$, where $\mathbb{F}(X)=\mathbb{F}(t)[\sqrt{h(t)}]$;

(c) if $\mathbb{F}(X)$ is imaginary, then so is $\mathbb{F}(Z)$; and

(d) there are maps $\operatorname{Jac}(X) \rightarrow \operatorname{Jac}(X) / H \hookrightarrow \operatorname{Jac}(Z)$, where $H$ is a finite group scheme annihilated by 2 .

Proof. Let $Y / \mathbb{F}$ be the hyperelliptic curve with branch locus $B \cup\{0, \infty\}$. Then the normalization $Z$ of the fiber product $X \times_{\mathbb{P}^{1}} Y$ is the sought-for curve [GP05, p. 305]. Now part (a) follows from loc. cit., part (b) records the fact that the branch locus of $Z \rightarrow \mathbb{P}_{s}^{1}$ is $\sqrt{B}$, and (d) follows from (a) and functoriality of the Picard functor. For (c), after an $\mathbb{F}$-linear change of coordinate on $\mathbb{P}^{1}$, we may assume that the point $t=1$ is in $B$, while $B$ is still disjoint from $\{0, \infty\}$. Then $s=1 \in \sqrt{B}$ is an F-rational branch point of $Z$.

Proof of Theorem 1. Let $X_{0} / \mathbb{F}_{q}$ be the smooth projective model of $K_{0}:=K$. Since $2 g\left(X_{0}\right)+2 \leq\left|\mathbb{P}^{1}\left(\mathbb{F}_{q}\right)-\{0, \infty\}\right|$, possibly after an $\mathbb{F}_{q}$-linear change of coordinate on $\mathbb{P}^{1}$, we assume that the branch locus $B_{0} \subset X_{0} /\langle\iota\rangle \cong \mathbb{P}^{1}$ is disjoint from $\{0, \infty\}$.

Using Lemma 2, inductively construct the hyperelliptic curve $X_{i}$ with branch locus $B_{i}=\sqrt{B_{i-1}}$. By Lemma2(d) and induction, if $N$ is an odd natural number, then $\operatorname{Jac}\left(X_{0}\right)[N]\left(\mathbb{F}_{q}\right)$ is a subgroup of $\operatorname{Jac}\left(X_{i}\right)[N]\left(\mathbb{F}_{q}\right)$ for each $i$.

Let $K_{i}$ be the function field $\mathbb{F}_{q}\left(X_{i}\right)$. Since $\operatorname{cl}\left(K_{i}\right) \cong \operatorname{Jac}\left(X_{i}\right)\left(\mathbb{F}_{q}\right), A \subset \operatorname{cl}\left(K_{i}\right)$ for each $i$. Moreover, if $K_{0}$ is imaginary, then each $K_{i}$ is as well (Lemma 2(c)).

Corollary 3. Let $h(t) \in \mathbb{F}_{q}[t]$ be a monic squarefree polynomial of even degree with $h(0) \neq 0$, and let $A \subset \operatorname{cl}\left(\mathbb{F}_{q}(t)[\sqrt{h(t)}]\right)$ be an abelian group of odd order. Then $A \subset \operatorname{cl}\left(\mathbb{F}_{q}(t)\left[\sqrt{h\left(t^{2^{i}}\right)}\right]\right)$ for all $i$.

Proof. Apply Lemma 2(b) to the construction in Theorem 1

Theorem 1 yields a short proof of some of the main results of [BJLS08, Pac09.

Example 4. Suppose 6 is invertible in $\mathbb{F}_{q}$. Let $c \in \mathbb{F}_{q}^{\times}$be a square, and let $E_{c}$ be the elliptic curve with affine model $y^{2}=x^{3}+c$; the three-division polynomial of $E_{c}$ is $x^{4}+4 c x$. Then $(0, \sqrt{c}) \in E_{c}\left(\mathbb{F}_{q}\right)$ is a point of order three, and there are infinitely many quadratic function fields over $\mathbb{F}_{q}$ with nontrivial 3-torsion in the class group. Now suppose that $q \equiv 1 \bmod 3$, and let $c=4^{2}$. Then $-4 c$ is a cube in $\mathbb{F}_{q}$ and $-3 c$ is a square, so that $E_{c}[3]\left(\mathbb{F}_{q}\right) \cong(\mathbb{Z} / 3)^{2}$. Therefore, there are infinitely many (imaginary) quadratic function fields over $\mathbb{F}_{q}$ with 3 -rank at least two.

Results about Newton polygons of curves are notoriously difficult to come by, even if one is willing to sacrifice control over the base field. The same fiber product construction sometimes allows one to amplify knowledge about Newton polygons of hyperelliptic curves over a given (finite) field.

Example 5. Suppose $p \equiv 2$ or $4 \bmod 7$. Then there are infinitely many hyperelliptic curves $X$ over $\mathbb{F}_{p}$ for which $1 / 3$ occurs in the Newton polygon of $\operatorname{Jac}(X)$. Indeed, let $X_{0} / \mathbb{F}_{p}$ be the hyperelliptic curve with affine model $y^{2}=x^{7}-1$. A special case of a calculation of Honda ([Hon66, p. 194]; see also [DH89, Cor. 2]) shows that the Newton polygon of $\operatorname{Jac}\left(X_{0}\right)$ is $\{1 / 3,2 / 3\}$. Inductively applying Lemma 2 , as in Theorem 1, produces a sequence of hyperelliptic curves $X_{i} / \mathbb{F}_{p}$ such that $\operatorname{Jac}\left(X_{i}\right)$ 
has a sub-abelian variety isogenous to $\operatorname{Jac}\left(X_{0}\right)$. Then $1 / 3$ is a slope of the Newton polygon of each $\operatorname{Jac}\left(X_{i}\right)$.

\section{ACKNOWLEDGMENTS}

It's a pleasure to acknowledge conversations with Rachel Pries and comments from the referee.

\section{REFERENCES}

[Ach06] Jeffrey D. Achter, The distribution of class groups of function fields, J. Pure Appl. Algebra 204 (2006), no. 2, 316-333. MR2184814 (2006h:11132)

[BJLS08] M. Bauer, M. J. Jacobson, Jr., Y. Lee, and R. Scheidler, Construction of hyperelliptic function fields of high three-rank, Math. Comp. 77 (2008), no. 261, 503-530 (electronic). MR2353964 (2008i:11135)

[DH89] Bert Ditters and Simen J. Hoving, On the connected part of the covariant Tate pdivisible group and the $\zeta$-function of the family of hyperelliptic curves $y^{2}=1+\mu x^{N}$ modulo various primes, Math. Z. 200 (1989), no. 2, 245-264. MR978298 (90e:14047)

[EVW09] Jordan Ellenberg, Akshay Venkatesh, and Craig Westerberg, Homological stability for Hurwitz spaces and the Cohen-Lenstra conjecture over function fields, December 2009, arXiv:0912.0325.

[FW89] Eduardo Friedman and Lawrence C. Washington, On the distribution of divisor class groups of curves over a finite field, Théorie des nombres (Quebec, PQ, 1987), de Gruyter, Berlin, 1989, pp. 227-239. MR.91e:11138

[GP05] Darren Glass and Rachel Pries, Hyperelliptic curves with prescribed p-torsion, Manuscripta Math. 117 (2005), no. 3, 299-317. MR2154252 (2006e:14039)

[Hon66] Taira Honda, On the Jacobian variety of the algebraic curve $y^{2}=1-x^{l}$ over a field of characteristic $p>0$, Osaka J. Math. 3 (1966), 189-194. MR0225777 (37:1370)

[Pac09] Allison M. Pacelli, Function fields with 3-rank at least 2, Acta Arith. 139 (2009), no. 2, 101-110. MR2539539

Department of Mathematics, Colorado State University, Fort Collins, Colorado 80523

E-mail address: j.achter@colostate.edu

URL: http://www.math. colostate.edu/ achter 